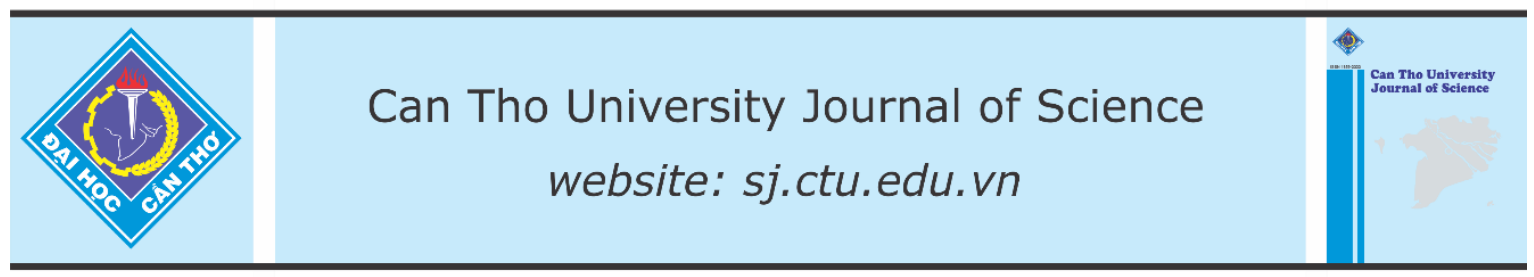

DOI: 10.22144/ctu.jen.2020.027

\title{
In vitro anti-inflammatory resorcinol derivatives and their in silico analysis
}

Rahen Mahmuda ${ }^{1}$, Shahenul Islam ${ }^{1}$, Negar Sultana Shoshi ${ }^{1}$, Khadija Akhter Poly ${ }^{1}$, Pranoy Saha ${ }^{1}$, Shamima Akhter ${ }^{1}$, Tran Quang De ${ }^{2}$ and Sukumar Bepary ${ }^{1 *}$

${ }^{1}$ Department of Pharmacy, Jagannath University, Dhaka-1100, Bangladesh

${ }^{2}$ Department of Chemistry, College of Natural Sciences, Can Tho University, Vietnam

*Correspondence: Sukumar Bepary (email: sukumarsb@yahoo.com)

\section{Article info.}

Received 11 Jun 2020

Revised 10 Sep 2020

Accepted 30 Nov 2020

\section{Keywords}

Anti-inflammatory, docking, nitrobenzoic acid, resorcinol

\begin{abstract}
Resorcinol with its two hydroxyl groups was derivatized in laboratory to observe the anti-inflammatory potential in vitro. Subsequently, in silico docking analysis was done for observing the binding modes in cyclooxygenase enzyme to have idea about the subsequent possible developments. At the doses of $200 \mu \mathrm{g} / \mathrm{mL}$ and $400 \mu \mathrm{g} / \mathrm{mL}$, the compounds showed the antiinflammatory property. Among them, 1,3-phenylene bis(2-chloro-4-nitrobenzoate) also offered dose dependent $51 \%$ and $70 \%$ of inhibition of heatinduced hemolysis respectively. The scaffold thus poses as an interesting pharmacophore suitable for further development for managing the inflammatory disorders.
\end{abstract}

Cited as: Mahmuda, R., Islam, S., Shoshi, N.S., Poly, K.A., Saha, P., Akhter, S., De, T.Q. and Bepary, S., 2020. In vitro anti-inflammatory resorcinol derivatives and their in silico analysis. Can Tho University Journal of Science. 12(3): 80-84.

\section{INTRODUCTION}

Cyclooxygenase (COX) is responsible for formation of prostanoids (Jane, 2019) including thromboxane and prostaglandins like prostacyclin, from arachidonic acid. The two types, namely COX-1 and COX-2 having minor variations in structure and distribution pattern in the human body, are responsible for different types of human chronic inflammatory disorders like cancer, cardiovascular diseases, diabetes, obesity, osteoporosis, rheumatoid arthritis, inflammatory bowel disease, asthma, depression and Parkinson's disease, etc. Though there are diversified classes of non-steroidal anti-inflammatory drugs (NSAIDs), most of them results in gastrointestinal complications (da Silva Guerra et al., 2011; dos Santos et al., 2012; Elhenawy et al., 2014; Suryawanshi et al., 2014, Goldstein et al.,
2015) including primary local irritation to gastrointestinal ulceration. These agents are not suitable for long term applications in managing the chronic inflammatory disorders, thereby demanding the better alternatives. Thus, this scaffold has been considered for study and the results have been reported here.

\section{EXPERIMENTAL SECTION}

\subsection{Materials and methods}

The necessary reagents were purchased from Sigma-Aldrich (USA) and TCI (Japan). Methanol and dichloromethane were collected from Duksan Pure Chemicals Co. Ltd, South Korea. Solvents were collected from Daejung Chemical \& Metal Co. Ltd. Derivatives were synthesized in the laboratory and then were purified by flash column chromatography using silica gel $(45-100 \mu)$. The reaction end 
points were checked by the TLC using Sigma-Aldrich Glass plates having silica gel coated with fluorescent indicator F254. The compounds were characterized by ${ }^{1} \mathrm{H}$ NMR by Bruker $400 \mathrm{MHz}$.

\subsection{Synthesis of resorcinol derivatives}

To the stirred solution of appropriate benzoic acid (A) was added 3.0 equivalent of thionyl chloride and the resulting solution was refluxed for 2 hours (Scheme-1). After subsequent removal of excess thionyl chloride by vacuum evaporation, dry dichloromethane was added. Under an ice bath system then were added triethylamine and resorcinol. Stirring was continued for overnight and reaction end point was confirmed by using of TLC. After subsequent addition of distilled water, the organic layer was collected by a separating funnel, washed successively by brine and water. The organic layer was then dried with sodium sulfate before it was subjected to filtration by using Whatman filter paper to collect the filtrate. The solvent was evaporated in vacuo to afford crude esters which were then purified by flash column chromatography to get the desired products (01-03, 82-88\% yield).<smiles>[R]c1cc([N+](=O)[O-])ccc1C(=O)O</smiles><smiles>[R]c1cc([N+](=O)[O-])ccc1C(=O)Oc1cccc(OC(=O)c2ccc([N+](=O)[O-])cc2[R])c1</smiles><smiles>[R]O[C@H](C)[C@H](C(=O)Oc1cccc(O)c1)c1ccc([N+](=O)[O-])cc1[R]</smiles>

Scheme 1: Synthesis of resorcinol derivatives

\subsection{Observation of the in vitro anti- inflammatory activity}

Anti-inflammatory activity was done by following the reported methods (Okoli et al., 2008) with some minor modifications. Isotonic buffer solution (5 $\mathrm{mL}$ ) was taken in centrifuge tubes containing 200 and/or $400 \mu \mathrm{g} / \mathrm{mL}$ of the test drugs. The centrifuge tubes were taken in 4 sets (per concentration). Tubes containing $5 \mathrm{~mL}$ of the vehicle only were taken for control. Reference standards were taken as 2 sets of $5 \mathrm{ml}$ buffer solution containing $100 \mu \mathrm{g} / \mathrm{mL}$ of ibuprofen. Erythrocyte suspensions $(0.05 \mathrm{~mL})$ were added to each tube. After gentle mixing, one set of the tubes was incubated at $54^{\circ} \mathrm{C}$ for 20 minutes and the other pair was maintained at $0-4^{\circ} \mathrm{C}$ in a freezer for 20 minutes. The reaction mixture was then centrifuged at 3,000 rpm for 3 minutes at the end of the incubation and absorbance (OD) of the supernatant was measured at $540 \mathrm{~nm}$ using isotonic buffer solution as the blank.
The level of hemolysis was calculated by using the following equation:

Percentage of hemolysis $=100 \times\left(\mathrm{OD}_{2}-\mathrm{OD}_{1}\right) /\left(\mathrm{OD}_{3}{ }^{-}\right.$ $\left.\mathrm{OD}_{1}\right)$

Where, $\quad \mathrm{OD}_{1}=$ absorbance of test sample unheated; $\mathrm{OD}_{2}=$ absorbance of test sample heated;

$\mathrm{OD}_{3}=$ absorbance of control sample heated.

The percentage inhibition of hemolysis was calculated by using the following relation:

Inhibition of hemolysis $(\%)=100-$ hemolysis $(\%)$

\subsection{Docking analysis}

The compounds were subsequently docked into the cyclooxygenase- 2 enzyme. The enzyme protein Data Bank (PDB) was collected from the internet (Orlando et al., 2015). The original PDB was having the reference drug ibuprofen docked into the ligand binding site of cyclooxygenase- 2 enzyme. However, after getting the original PDB file, the ligand 
was separated out and the receptor PDB was processed for the docking studies.

\subsubsection{Preparation of the ligand file of the synthesized compounds}

The compound structures were drawn by using the ChemDraw software. The structures thus obtained were in 2-D from and thus were converted to 3-D which were then transformed to PDBQT files by using the Python Molecular Viewer (PMV).

\subsubsection{Docking of the ligand PDBQT in the receptor PDBQT file}

The ligand PDBQT files were then docked in the receptor PDBQT by using the Autodock Vina software (Trott et al., 2010). The output PDBQT files were then viewed by using the PyMOL software. The binding modes with lowest energy were taken under consideration for interaction analysis. This selected ligand PDBQT files were taken along with the ligand PDB to find out the binding interactions and next possible improvements.

\section{RESULTS AND DISCUSSION}

\subsection{Synthesis of resorcinol ester of benzoic acids}

To the benzoic acid, thionyl chloride, triethylamine, resorcinol, and other necessary chemicals, reagents and utilities were collected from local suppliers. The laboratory condition was sufficient for getting very good yields. The process was reproducible and thus can be used for further derivatizations if desired. Compounds were characterized by using the ${ }^{1} \mathrm{H}$ NMR available in the Bangladesh Council of Scientific and Industrial Research (BCSIR).

\subsection{Proton NMR data of the synthesized compounds}

Comound (01) 1,3-Phenylene bis(4-nitrobenzoate) is an orange solid with yield of $77 \%$ and ${ }^{1} \mathrm{H}$ NMR (400 MHz, $\left.\mathrm{CDCl}_{3}\right): \delta$ 7.20-7.24 (m, 2H), $7.54(\mathrm{t}, J$ $=8.0 \mathrm{~Hz}, 1 \mathrm{H}), 8.26-8.39(\mathrm{~m}, 9 \mathrm{H})$.

Comound (02) 1,3-Phenylene bis(2-chloro-4-nitrobenzoate) is a pale orange solid with yield of $81 \%$ and ${ }^{1} \mathrm{H}$ NMR (400 MHz, $\left.\mathrm{CDCl}_{3}\right): \delta 7.23-7.29(\mathrm{~m}$, $3 \mathrm{H}), 7.54(\mathrm{t}, J=8.0 \mathrm{~Hz}, 1 \mathrm{H}), 8.17-8.20(\mathrm{~m}, 2 \mathrm{H})$, 8.23-8.25 (m, 2H), 8.39 (m, 2H).

Comound (03) 3-Hydroxyphenyl 2-chloro-4-nitrobenzoate is a yellow solid with yield of $79 \%$ and ${ }^{1} \mathrm{H}$ NMR (400 MHz, $\left.\mathrm{CDCl}_{3}\right): \delta 6.76-6.82(\mathrm{~m}, 3 \mathrm{H}), 7.28$ $(\mathrm{t}, J=8.0 \mathrm{~Hz}, 1 \mathrm{H}), 8.13-8.15(\mathrm{~m}, 1 \mathrm{H}), 8.21-8.26(\mathrm{~m}$, $1 \mathrm{H}), 8.37-8.38(\mathrm{~m}, 1 \mathrm{H})$.

\subsection{Biological evaluation of resorcinol ester of benzoic acids}

The compounds were observed for the anti-inflammatory property by using the in vitro methods where the efficacy in preventing the heat induced hemolysis of the human red blood cells (HRBC) was observed. The results obtained have been given in the Table 1.

Table 1: Efficacy of compound 01,02 and 03 in preventing the heat induced hemolysis

\begin{tabular}{lrrrrrr}
\hline \multirow{2}{*}{ Sample } & $\begin{array}{r}\text { Strength } \\
(\boldsymbol{\mu} \mathbf{g} / \mathbf{m l})\end{array}$ & \multicolumn{2}{c}{ Hot } & Cold & $\begin{array}{r}\text { Lysis (\%) } \\
\text { (Heat-Cool)/(Control- } \\
\text { Cool)*100 }\end{array}$ & $\begin{array}{r}\text { Inhibition (\%) } \\
\text { (100-Lysis\%) }\end{array}$ \\
\hline 01 & 200 & 0.060 & 0.048 & 44 & 56 \\
02 & 200 & 0.052 & 0.031 & 49 & 51 \\
03 & 400 & 0.043 & 0.029 & 30 & 70 \\
Ibuprofen & 200 & 0.064 & 0.038 & 72 & 27 \\
Control & 100 & 0.038 & 0.030 & 17 & 83 \\
\hline
\end{tabular}

The compound $\mathbf{0 3}$ was found to be comparatively weak (27\% inhibition) in preventing the lysis of HRBC. Whereas, compound $\mathbf{0 2}$ having di-substitution, showed significantly higher inhibition (51\%). There was a good dose-depended increase in the efficacy as shown by $400 \mu \mathrm{g} / \mathrm{mL}$ (70\% inhibition). The compound $\mathbf{0 1}$, having di-substitution but no halogen, was comparable with 02. Ibuprofen was used as the reference compound in this study.

\subsection{In silico analysis for the binding pattern}

Docking analysis was done by using the Autodock Vina software published from The Scriffs Research Institute. Compound $\mathbf{0 1}$ and $\mathbf{0 2}$ showed similar types of inhibition and so the former was taken for this study. In this study, various modes were observed from the output PDBQT files having different energy levels. The lowest energy mode was taken into consideration for further prediction. 

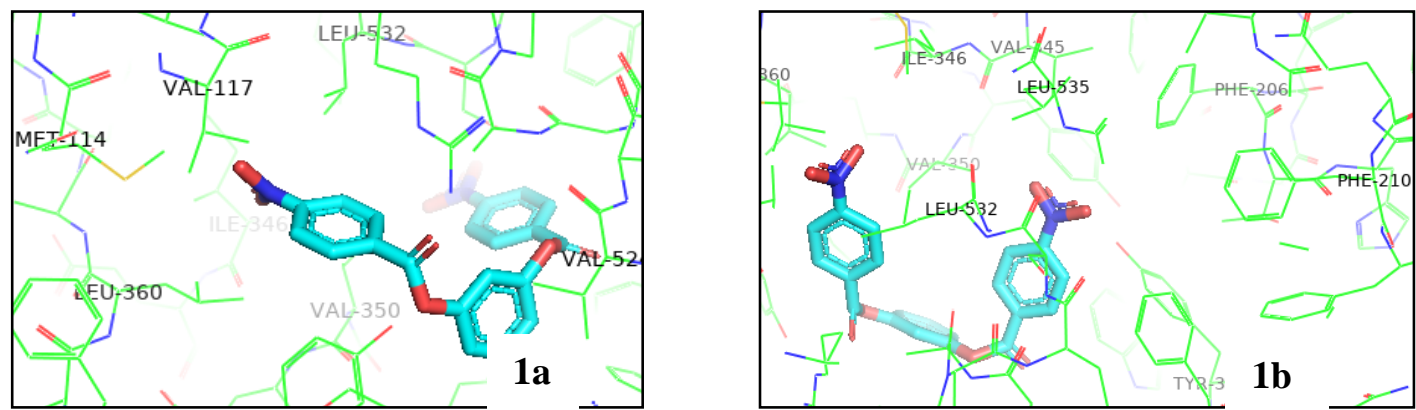

Figure 1: Projection of nitrobenzene groups to a non-polar pocket of the COX enzyme

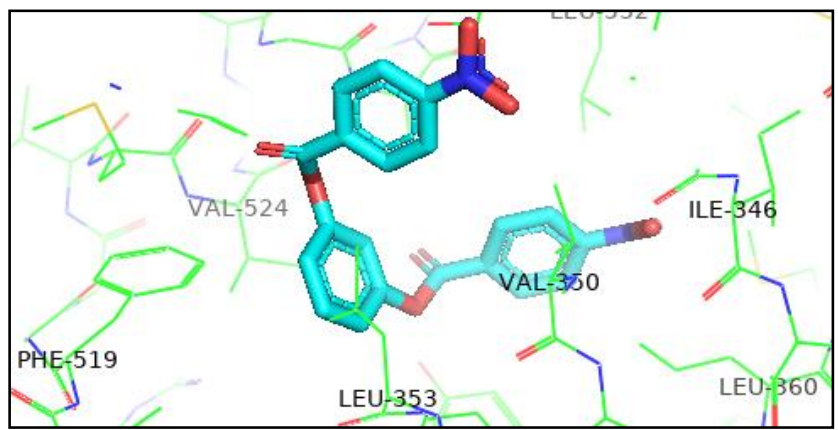

Figure 2: Access of the central phenyl to a non-polar site of the COX enzyme

One of the nitrobenzene moieties was found to be projected to a side pocket having the non-polar characters due to the dominance of the side chains from Val-117, Met-114, Leu-532, and Leu-360 as shown in Figure 1. Thus more groups can be tried based on this study. The other nitrobenzene ring was also found to be projected to another non-polar site though was staying little far. Accordingly, this ring can be replaced to ensure proper fit in this pocket. On the other hand, the central phenyl ring has projected its unsubstituted part to another site having non-polar residues like, Phe-529, Val-523, Leu-353. But as shown in Figure 2, there is little more blank space which can be utilized for introducing additional non-polar groups and/or atoms on this central ring.

\section{CONCLUSIONS}

It is obvious that the resorcinol derivatives possessed encouraging potency for inhibiting the heatinduced lysis of the HRBC membrane which may be significant while targeting the chronic inflammatory disorders. Moreover, since this scaffold does not bear any carboxylic acid group, this may be better tolerated option for the long-term managements. Thus further study should be done with this scaffold to develop more derivatives and thereby to make a comprehensive structure-activity-relationship study with this pharmacophore.

\section{ACKNOWLEDGMENTS}

We are thankful to Jagannath University and University Grants Commission of Bangladesh for providing the required laboratory facilities and complementary financial supports for this project.

\section{REFERENCES}

da Silva Guerra, A. S. H., do Nascimento Malta, D. J., Laranjeira, L. P. M., et al., 2011. Anti-infammatory and antinociceptive activities of indole-imidazolidine derivatives. Int. Immunopharmacol., 11(11): 18161822. DOI 10.1016/j.intimp.2011.07.010.

dos Santos, J. L., Moreira, V., Campos, M. L., et al., 2012. Pharmacological evaluation and preliminary pharmacokinetics studies of a new diclofenac prodrug without gastric ulceration effect. Int. J. Mol. Sci. 13(11): 15305-15320. DOI 10.3390/ijms131115305.

Elhenawy, A. A., El-Gazzar, M. A., and Mohmmed, H. M., 2014. Synthesis, anti-Infammatory, analgesic, molecular modeling and ADMET studies of novel diclofenac derivatives containing alanyl moiety. Chemistry and Materials Research. 6(2): 69-77. DOI doi.org/10.7176/CMR 
Goldstein, J. L. and Cryer, B., 2015. Gastrointestinal injury associated with NSAID use: a case study and review of risk factors and preventative strategies. Drug Healthc Patient Saf. 7: 31-41. DOI 10.2147/DHPS.S71976.

Jane, A. M. and Nicholas, S. K., 2019. Eicosanoids, prostacyclin and cyclooxygenase in the cardiovascular system. Br. J. Pharmacol. 176: 1038-1050. DOI 10.1111/bph.14167.

Okoli, C. O., Akah, P. A., Onuoha, N. J., Okoye, T. C., Nwoye, A. C. and Nworu, C. S., 2008. Acanthus montanus: An experimental evaluation of the antimicrobial, anti-inflammatory and immunological properties of a traditional remedy for furuncles. BMC Complementary and Alternative Medicine.8: 27. DOI 10.1186/1472-6882-8-27.
Orlando, B. J., Lucido, M. J. and Malkowski M. G., 2015. The structure of ibuprofen bound to cyclooxygenase-2. J. Struct. Biol. 189: 62. DOI 10.2210/pdb4PH9/pdb.

Suryawanshi, S. B., Osman, H. A., Shaikh, Y. I. and Nazeruddin, G. M., 2014. Synthesis of Various esters of diclofenac (NSAIDs) as pro-drugs and their biological evaluation. Chemical Science Transactions. 3: 562-565. DOI 10.7598/cst2014.745.

Trott, O. and Olson, A. J., 2010. AutoDock Vina: Improving the speed and accuracy of docking with a new scoring function, efficient optimization, and multithreading, J. Comput. Chem. 31(2): 455-461. DOI $10.1002 /$ jcc. 21334 . 